\title{
La SFP aujourd'hui. Où aller, pour qui et avec qui ?
}

\section{Allocution du président entrant, présentée à l'assemblée générale du 5 février 2016}

Je vous remercie pour la confiance que vous m’avez témoignée en m'élisant à la tête de la Société Française de Physique. Je m'inscrirai dans la continuité de l'action du président sortant Alain Fontaine, que je remercie pour son dynamisme et pour tout ce qu'il a fait. La force de notre association vient de ses près de 3000 adhérents, elle s'exprime à travers les actions de terrain de ses sections locales et les travaux de ses commissions, elle se structure par son organisation en divisions thématiques. L'assemblée générale, le Conseil d'administration, le bureau et le siège de la SFP cadrent et accompagnent l'ensemble des activités. La Société Française de Physique, c'est aussi une histoire de près de 150 ans qui la place au cœur de la communauté des physicien.ne.s et dont elle tire une légitimité forte.

Une communauté et une histoire au service des sciences et plus particulièrement de la physique, inspirées du siècle des Lumières

Par ses colloques, son congrès, ses prix, sa revue Reflets de la physique, la SFP contribue à animer la vie scientifique et à structurer la communauté des physicien.ne.s.

Organisatrice et soutien de nombreuses activités tournées vers le grand public, les jeunes et l'accompagnement des enseignants, la SFP ambitionne de sensibiliser à l'importance de la démarche scientifique dans la construction et le partage des connaissances, et à l'importance de la physique comme moteur des innovations qui transforment la société.

\section{Les valeurs}

Elles sont au cœur de notre activité. Ce sont, entre autres :

- la créativité et l'exigence de la démarche scientifique, associées à l'intégrité et à l'éthique nécessaires dans le travail scientifique ;

- la reconnaissance de la diversité comme un atout pour nos activités ;

- la collaboration et une science citoyenne.

\section{Les prises de position}

Animés de ces valeurs, nous prenons part aux sujets qui nous concernent au sein du débat public, comme :

- le soutien à l'accès libre et gratuit aux publications à travers une aide institutionnelle, notamment à l'éditeur EdP Sciences dont nous sommes le principal actionnaire ;

- I'évaluation multicritères, par les pairs, des chercheurs, des publications et des laboratoires ;

- I'assistance à un enseignement participatif des sciences, en équipes, passant par la découverte : ainsi La main à la pâte en primaire, les Olympiades de Physique au lycée, I'International Physicists' Tournament dans le supérieur...

- la valorisation du diplôme de docteur auprès des entreprises ;

- I'égalité des chances entre hommes et femmes en physique ;

- I'affichage de la position de la Société Européenne de Physique (EPS) sur le sujet de l'énergie, position que la SFP a grandement contribué à forger.

\section{Les relations avec les autres sociétés savantes}

La Société Française de Physique s'honore de relations très suivies avec les autres sociétés savantes françaises (notamment la Société Française d'Optique et la Société Chimique de France), avec la Société Européenne de Physique, et avec les sociétés sœurs allemande, britannique, italienne, suisse et américaine. Elle souhaite aussi renforcer ses relations avec l'Académie des sciences, en vue d'actions et de prises de positions communes.

Pour conclure, notre force c'est vous. La tâche est immense pour mieux faire avancer, connaître et partager la science et ses valeurs. C'est un défi considérable pour nous tous, dans une société qui se transforme en profondeur.

Michel Spiro Président de la Société Française de Physique 